\title{
The Evolution of the Angular Momentum Distribution during Star Formation
}

\author{
Kohji Tomisaka ${ }^{1}$ \\ Faculty of Education and Human Sciences, Niigata University, 8050 Ikarashi-2, Niigata \\ 950-2181
}

Received August 18, 1999; accepted October 28, 1999

\footnotetext{
${ }^{1}$ E-mail: tomisaka@ed.niigata-u.ac.jp
} 


\begin{abstract}
If the angular momentum of the molecular cloud core were conserved during the star formation process, a new-born star would rotate much faster than its fission speed. This constitutes the angular momentum problem of new-born stars. In this paper, the angular momentum transfer in the contraction of a rotating magnetized cloud is studied with axisymmetric MHD simulations. Owing to the large dynamic range covered by the nested-grid method, the structure of the cloud in the range from $10 \mathrm{AU}$ to $0.1 \mathrm{pc}$ is explored. First, the cloud experiences a run-away collapse, and a disk forms perpendicularly to the magnetic field, in which the central density increases greatly in a finite time-scale. In this phase, the specific angular momentum $j$ of the disk decreases to $\simeq 1 / 3$ of the initial cloud. After the central density of the disk exceeds $\sim 10^{10} \mathrm{~cm}^{-3}$, the infall on to the central object develops. In this accretion stage, the rotation motion and thus the toroidal magnetic field drive the outflow. The angular momentum of the central object is transferred efficiently by the outflow as well as the effect of the magnetic stress. In $7000 \mathrm{yr}$ from the core formation, the specific angular momentum of the central $0.17 M_{\odot}$ decreases a factor of $10^{-4}$ from the initial value (i.e. from $10^{20} \mathrm{~cm}^{2} \mathrm{~s}^{-1}$ to $10^{16} \mathrm{~cm}^{2} \mathrm{~s}^{-1}$ ).
\end{abstract}

Subject headings: ISM: clouds - ISM: jets and outflows - ISM: magnetic fields stars: formation - stars: rotation 


\section{INTRODUCTION}

The problem of star formation has been the object of serious study for many years. In the star formation process, almost all the angular momentum should be

removed from the molecular cloud in the course of forming stars. For example, using rotation periods and the radius of classical T Tauri stars (CTTS) $[P=3-10$ days and $R_{*} \simeq 2 R_{\odot}$ (Bouvier et al. 1993)], the specific angular momentum of such stars is approximately equal to $j \simeq 4 \pi R_{*}^{2} /(5 P) \sim 5.6 \times 10^{16} \mathrm{~cm}^{2} \mathrm{~s}^{-1}\left(R_{*} / 2 R_{\odot}\right)^{2}(P / 10 \text { day })^{-1}$, where we assumed the star rotates uniformly. On the other hand, if the velocity gradient of $0.3 \mathrm{~km} \mathrm{~s}^{-1} \mathrm{pc}^{-1}-4 \mathrm{~km} \mathrm{~s}^{-1} \mathrm{pc}^{-1}$ (Goodman et al. 1993) observed in $\mathrm{NH}_{3}$ cores (density $\gtrsim 10^{4} \mathrm{~cm}^{-3}$; size $\sim 0.1 \mathrm{pc}$ ) comes from the rotation, $j$ reaches $\sim 5 \times 10^{21} \mathrm{~cm}^{2} \mathrm{~s}^{-1}(R / 0.1 \mathrm{pc})^{2}\left(\Omega / 4 \mathrm{~km} \mathrm{~s}^{-1} \mathrm{pc}^{-1}\right)$. Therefore, the specific angular momentum should decrease a factor of $10^{-5}$ when a star is formed from a molecular cloud core. Assuming $j$ is conserved through the collapse, the gravity is balanced with the centrifugal force at the centrifugal radius as $R_{c}=j^{2} /(G M) \sim 0.06 \mathrm{pc}\left(j / 5 \times 10^{21} \mathrm{~cm}^{2} \mathrm{~s}^{-1}\right)^{2}\left(M / 1 M_{\odot}\right)^{-1}$. This leads to the conclusion that if the angular momentum is not efficiently reduced or transferred, the cloud could not shrink to become a stellar object $\left(R_{c} \gg R_{*}\right)$. This is called the angular momentum problem for new-born stars.

To reduce the spin angular momentum of newly formed stars, two mechanisms are proposed. One is to transfer the cloud spin angular momentum to the orbital angular momentum of binary or multiple protostars if they are made by fission. The other possibility is magnetic braking, in which angular momentum is transferred to the ambient medium by the magnetic stress (Spitzer 1978). The latter works even for the case of a single star. In the case of the parallel rotator, in which the directions of magnetic fields and angular momentum coincide with each other, the time scale of deceleration of the angular rotation 
speed $(\Omega)$ is given by

$$
t_{B} \equiv-\frac{\Omega}{d \Omega / d t} \simeq \frac{\sigma}{2 \rho_{a} V_{A}}=\frac{2 \pi G \sigma}{B_{0}}\left(4 \pi G \rho_{a}\right)^{-1 / 2},
$$

where $\sigma, \rho_{a}, V_{A}$, and $G$ denote, respectively, the column density of the molecular core, the ambient density, the Alfvén speed of the ambient medium, and the gravitational constant (Ebert, von Hoerner, \& Temesvary 1960, Mouschovias, \& Paleologou 1980). As long as the molecular core evolves in a quasistatic manner, the decrease in the angular momentum $L$ is well fitted by the above time scale as $L=L(t=0) \exp \left(-t / t_{B}\right)$ (Tomisaka, Ikeuchi, \& Nakamura 1990). Since the first factor $2 \pi G \sigma / B_{0}$ is approximately equal to or larger than unity for a gravitationally contracting cloud (Tomisaka 1995), $t_{B}$ is longer than the free-fall time of the ambient low-density medium. This raises the question of whether or not the angular momentum is efficiently transferred while the contraction proceeds.

Recently, bipolar outflows have been found in a wide variety of objects which indicate star formation (for a review, see Bachiller 1996). Based on the magnetic acceleration model of outflow (Blandford, \& Payne 1982; see also Königl 1989 and Wardle, \& Königl 1993), magnetic fields extract the angular momentum from the disk and transfer it to the outflow gas. Therefore, the outflow gas is able to carry the excess angular momentum directly.

From MHD simulations of the gravitational contraction of a magnetized, rotating isothermal molecular cloud, it is shown that the outflow begins after the central density exceeds $10^{10} \mathrm{~cm}^{-3}$ and an adiabatic core forms in the center (Tomisaka 1998, hereafter Paper I). The outflow is never driven in the run-away collapse phase, since rotation motion and thus toroidal magnetic fields are unimportant in this phase. Therefore, in this paper, we study the evolution of the cloud from the runaway collapse phase to the accretion phase, continuously. In particular, we try to determine when the redistribution of angular momentum occurs and whether the outflow plays an important role in extracting the angular momentum from the contracting gas. The model and the numerical method are 
described in $\S 2$. In $\S 3$, the evolution of the specific angular momentum distribution is

shown. In addition, a comparison is made between the effects of the magnetic torque and outflow to reduce the angular momentum. We discuss the ideal MHD condition and the evolution of late stage in $\S 4$.

\section{Model and Numerical Method}

We began our simulation from an infinitely long, cylindrical, rotating, isothermal cloud in hydrostatic balance. In terms of the gravitational potential, $\psi_{0}$, the magnetic flux density, $\mathbf{B}_{0}=B_{z} \mathbf{e}_{z}$, the angular rotation speed, $\Omega_{0}$, and the density $\rho_{0}$, the hydrostatic configuration is governed by the equations

$$
\begin{gathered}
r \Omega_{0}^{2}-\frac{d \psi_{0}}{d r}-\frac{c_{s}^{2}}{\rho_{0}} \frac{d \rho_{0}}{d r}-\frac{1}{8 \pi} \frac{d B_{z}^{2}}{d r}=0, \\
\frac{1}{r} \frac{d}{d r}\left(r \frac{d \psi_{0}}{d r}\right)=4 \pi G \rho_{0} .
\end{gathered}
$$

These equations have a solution for the radial distributions of density, rotational velocity, and magnetic flux density as follows (Stodółkiewicz 1963):

$$
\begin{gathered}
\rho_{0}(r)=\rho_{c}\left[1+r^{2} /\left(8 H^{2}\right)\right]^{-2}, \\
v_{\phi}(r) \equiv r \Omega_{0}(r)=r \Omega_{c}\left[1+r^{2} /\left(8 H^{2}\right)\right]^{-1 / 2}, \\
B_{z}(r)=B_{c}\left[1+r^{2} /\left(8 H^{2}\right)\right]^{-1},
\end{gathered}
$$

where $H$ represents the scale-height as $4 \pi G \rho_{c} H^{2}=c_{s}^{2}+B_{c}^{2} /\left(8 \pi \rho_{c}\right)+2 \Omega_{c}^{2} H^{2}$ [for $\left.\Omega_{c}<\left(2 \pi G \rho_{c}\right)^{1 / 2}\right]$. Here, quantities with a subscript $\mathrm{c}$ denote those for the cloud center $(r=0)$. This hydrostatic solution contains two nondimensional parameters: $\omega \equiv \Omega_{c} /\left(4 \pi G \rho_{c}\right)^{1 / 2}$ and $\alpha \equiv B_{c}^{2} /\left(4 \pi \rho_{c} c_{s}^{2}\right)$.

We assume that the gas obeys the ideal MHD equations. The gas is assumed isothermal for low densities. However, after the density exceeds $\sim 10^{10} \mathrm{~cm}^{-3}$, the interstellar gas is 
not efficiently cooled and behaves adiabatically (Larson 1969). To mimic this situation, a barotropic relation such as

$$
p\left\{\begin{array}{l}
=c_{s}^{2} \rho, \ldots\left(\rho<\rho_{\text {crit }}\right) \\
=c_{s}^{2} \rho_{\text {crit }}\left(\rho / \rho_{\text {crit }}\right)^{\Gamma}, \ldots\left(\rho>\rho_{\text {crit }}\right)
\end{array}\right.
$$

was adopted without using the energy equation, where we take $\rho_{\text {crit }}=10^{10} \mathrm{H}_{2} \mathrm{~cm}^{-3}$. The polytropic exponent $\Gamma$ was taken as $5 / 3$ and 2. (The specific heat ratio $\gamma$ never exceeds $5 / 3$. However, we found that the flow outside the core and the mass-accretion rate are essentially the same irrespective of the values of the core's polytropic exponent, $\Gamma$. Therefore, we used the larger exponent to reduce the effort to perform numerical computations.) To initiate gravitational contraction, we added density perturbation with small amplitude. The periodic boundary condition was applied to the boundaries in the $z$-direction, and the separation between two boundaries was chosen to be identical with the wavelength of the gravitationally most unstable mode (Matsumoto, Hanawa, \& Nakamura 1997). To ensure the fine spatial resolution necessary especially near the forming adiabatic core, the nested-grid method was applied to the MHD finite difference scheme (Tomisaka 1996a, 1996b, 1998). In the nested-grid method, 15 levels of grids are used from L0 (the coarsest) to L14 (the finest). A grid spacing of $\mathrm{L} n$ was chosen as $1 / 2$ of that of $\mathrm{L} n-1$, and each level of grids was divided into $64 \times 64$ cells. The code was tested by comparing the result of gravitational collapse calculated by a code without the nested-grid method (for details, see Tomisaka 1996b).

\section{RESULTS}

Here, the results of the model with $\omega=1 / 2$ and $\alpha=1$ are shown. This corresponds to $\Omega=2.78 n_{c 4}^{1 / 2} \mathrm{~km} \mathrm{~s}^{-1} \mathrm{pc}^{-1}$ and $B_{c}=13.3 n_{c 4}^{1 / 2} c_{s 190} \mu \mathrm{G}$. Here, $n_{c 4}$ and $c_{s 190}$ represent respectively $\rho_{c} / 10^{4} \mathrm{H}_{2} \mathrm{~cm}^{-3}$ and $c_{s} / 190 \mathrm{~m} \mathrm{~s}^{-1}$. The parameters are taken to be the same as 
the model shown in Paper I. Evolutions are also the same.

\subsection{Specific Angular Momentum Distribution}

In Figure 1, the specific angular momentum $j$ is plotted against the mass measured from the center. Since the density increases monotonically reaching the center, a mass shell with higher density is considered to be located nearer to the center than that with lower density. The total mass of the gas whose density is higher than $\rho_{1}$,

$$
M\left(>\rho_{1}\right)=\int_{\rho>\rho_{1}} \rho(z, r) d V
$$

and the correspondent total angular momentum,

$$
L\left(>\rho_{1}\right) \equiv \int_{\rho>\rho_{1}} \rho(z, r) v_{\phi} r d V
$$

are calculated at three epochs. Figure 1 is made by plotting $j\left(>\rho_{1}\right) \equiv L / M$ against $M\left(>\rho_{1}\right)$ for various $\rho_{1}$. This is similar to the specific angular momentum spectrum (e.g. Fig.5 of Norman, Wilson, \& Barton 1980) in which $M(<j)$, the total mass whose specific angular momentum is smaller than $j$ is plotted against $j$. However, the plot in Figure 1 has an advantage that the gas with smaller $M$ is necessarily located nearer to the center.

Open circles represent the $j(<M)$ distribution in the early phase [ $\left.t=5.214\left(4 \pi G \rho_{c}\right)^{-1 / 2} \sim 0.9 n_{c 4}^{-1 / 2} \mathrm{Myr}\right]$, in which the central density increases a factor 10 from the initial state (i.e., $\rho_{c}$ reaches $\simeq 10^{5} n_{c 4} \mathrm{H}_{2} \mathrm{~cm}^{-3}$ ). At this stage, the density distribution is almost spherical. Filled squares denote the distribution at the end of the

run-away collapse phase $\left(t<5.980\left(4 \pi G \rho_{c}\right)^{-1 / 2} \sim 1.05 n_{c 4}^{-1 / 2} \mathrm{Myr}\right.$; the structure at the stage is shown in Fig. 2a of Paper I). Since the motion crossing the magnetic fields is blocked, a disk running perpendicularly to the magnetic fields is formed. From Figure 1, it is shown that the angular momentum contained in the central $\sim 0.5 c_{s 190}^{3} n_{c 4}^{-1 / 2} M_{\odot}$ is reduced to $\simeq 1 / 3$ before a core is formed. 
This reduction is not owing to the angular momentum transfer by the magnetic fields but to the change of the shape of high-density region (from spherical to disk-like). This is confirmed by the fact that the specific angular momentum spectrum (not shown in this Letter) is not changed during the run-away collapse phase. The disk is mainly formed by the gas flow parallel to the magnetic fields (z-direction). Therefore, central part of the run-away collapsing disk is made by a gas with small angular momentum sitting initially near the $z$-axis. In other words, to the high-density region the mass is preferentially gathered rather than the angular momentum. The difference between open circles and filled squares in Figure 1 is owing to the segregation of low-angular momentum gas in the run-away collapse stage. However, the amount of the angular momentum segregation is too insufficient to explain the angular momentum problem.

The specific angular momentum, $j$, is approximately proportional to the accumulated mass, $M$. This relation coincides with the prediction for the thin disk by Basu(1998). This relation is explained from the solution of the contracting isothermal thin disk, i.e., the column density $\sigma \simeq c_{s} / G r$ and the angular rotation speed $\Omega \propto r^{-1}$ (Matsumoto et al. 1997) lead to the specific angular momentum $j=\Omega r^{2} \propto r \propto M \equiv \int \sigma r d r$.

After the central density of the disk exceeds $\rho_{\text {crit }} \sim 10^{10} \mathrm{H}_{2} \mathrm{~cm}^{-3}$, a new phase appears: the accretion phase. Thermal photons emitted from the dust which cooled the cloud become optically thick; as a result, an almost spherical adiabatic core is formed (in the adiabatic core, the thermal pressure becomes more important). Gas around the core begins to accrete on the core.

As shown in Paper I, after $\sim 10^{3}$ yr has passed, 2 the outflow begins (Figures $2 \mathrm{~b}$ and 3 of Paper $\mathrm{I}$ ). The size of the seeding region (origin of the outflow) is

\footnotetext{
2 This age is independent on the values of the assumed polytropic index, $\Gamma=5 / 3$ and
} 2. Furthermore, the mass outflow rate differs slightly: $\Gamma=2$ model shows approximately 20 
$r \sim 5 \times 10^{-3} c_{s} /\left(4 \pi G \rho_{c}\right)^{1 / 2} \sim 35 c_{s 190} n_{c 4}^{-1 / 2} \mathrm{AU}$. The seeding region expands radially outward This outflow is driven by the gradient of the magnetic pressure of the toroidal magnetic fields, $-\nabla B_{\phi}^{2} / 8 \pi$, which are made by the rotation motion (Paper I;Kudoh, Matsumoto, \& Shibata 1998). The magnetic fields exert torque on the outflowing gas to increase its angular momentum. On the other hand, they exert torque on the disk to decrease the angular momentum (Blandford, \& Payne 1982). In $\tau=0.04\left(4 \pi G \rho_{c}\right)^{-1 / 2} \sim 7000 n_{c 4}^{-1 / 2}$ yr (filled triangles; $\tau$ is the time elapsed from the core formation epoch), the outflow expands and reaches $z \sim 0.25 c_{s}\left(4 \pi G \rho_{c}\right)^{-1 / 2} \sim 1800 c_{s 190} n_{c 4}^{-1 / 2} \mathrm{AU}$. The angular momentum distribution at that time is shown by the solid line in Figure 1. It is shown that the specific angular momentum contained in the central $\sim 0.17 c_{s 190}^{3} n_{c 4}^{-1 / 2} M_{\odot}\left(\rho>10^{10} n_{c 4} \mathrm{H}_{2} \mathrm{~cm}^{-3}\right)$ has been reduced to a factor $\lesssim 10^{-4}$ of the initial value.

\subsection{Angular Momentum Flux and Magnetic Torques}

Consider the total angular momentum, $L(<M)$, contained in the mass $M$. The increase and decrease of the angular momentum are caused by (1) the inflow (accretion flow) mainly in the disk $d L /\left.d t\right|_{\text {in }}$, (2) the torque exerted on the gas disk from the magnetic fields $N$, and (3) the outflow from the disk $d L /\left.d t\right|_{\text {out }}: d L / d t=d L /\left.d t\right|_{\text {in }}+d L /\left.d t\right|_{\text {out }}+N$. It is to be noted that only the first term is positive. To see which term is important to reduce

$\%$ smaller outflow rate than the model of $\Gamma=5 / 3$. However, in the case of slowly rotating cloud as $\alpha=1$ and $\omega=1 / 10$, which leads to a smaller centrifugal radius, in a model assuming $\Gamma=2$ the outflow begins earlier than than that of $\Gamma=5 / 3$. This seems to indicate that unless the centrifugal radius from which the outflow is mainly ejected is formed near the center, especially, in the hard core, assuming such a relatively hard polytropic index is justified. Quantitative comparison will be made in a forthcoming paper. 
the angular momentum, we plot these three quantities for the three epochs in Figure 2. To calculate $d L /\left.d t\right|_{\text {in }}$ and $d L /\left.d t\right|_{\text {out }}$, we consider a cylinder which covers the isodensity surface. The $d L /\left.d t\right|_{\text {out }}$ is measured by the angular momentum flux convected outwardly through the upper and lower surfaces of the cylinder, and $d L /\left.d t\right|_{\text {in }}$ is the flux running radially inward through the side surface.

Before the core formation (circles and squares), the angular momentum inflow $\left(d L /\left.d t\right|_{\text {in }}\right)$ is larger than the magnetic torque $(N)$ which reduces the angular momentum. (This does not mean that the specific angular momentum increases. Note that $d M / d t_{\text {in }}$ is also positive.) In contrast, in the accretion phase after outflow blows, the magnetic torque (filled triangles) is comparable with $d L /\left.d t\right|_{\text {in }}$ (open triangles) near $M \gtrsim 0.17 c_{s 190}^{3} n_{c 4}^{-1 / 2} M_{\odot}$ $\left(\tau=7000 n_{c 4}^{-1 / 2} \mathrm{yr}\right)$. Further, from the figure, it is shown that the angular momentum transport by outflow $d L /\left.d t\right|_{\text {out }}$ (open stars) is more efficient than that of the magnetic torque. By these two effects, the angular momentum transferred by the inflow inside the disk is totally removed for central $\sim 0.17 c_{s 190}^{3} n_{c 4}^{-1 / 2} M_{\odot}$. As a result, $j\left(<0.17 c_{s 190}^{3} n_{c 4}^{-1 / 2} M_{\odot}\right)$ is reduced a factor of $10^{-4}$ from the initial value.

\section{DISCUSSION}

The size of the outflow reaches $z \sim 0.25 c_{s} /\left(4 \pi G \rho_{c}\right)^{1 / 2} \sim 1800 c_{s 190} n_{c 4}^{-1 / 2} \mathrm{AU}$ in $\tau \sim 7000 n_{c 4}^{-1 / 2}$ yr. However, CO observations show that the molecular outflows have typical spatial size of $8000 \mathrm{AU}-4$ pc (Fukui et al. 1993). This simulation is limited in time and should be extened further. However, calculation becomes harder after the core mass exceeds $\sim 0.1 c_{s 190}^{3} n_{c 4}^{-1 / 2} M_{\odot}$ due to high density at the cloud center. Let us consider whether the magnetic angular momentum transport is effective still in the later stage $\tau>7000 n_{c 4}^{-1 / 2} \mathrm{yr}$.

As shown in Figure 1, $j$ is approximately proportional to $M$ at the epoch of the 
core formation. Writing down the relation as $j=p \times G M / c_{s}$, the coefficient $p$ is approximately equal to 0.1 for the model shown in Figure 1 . If $j$ is conserved, the centrifugal radius $\left(R_{c}\right)$, at which the gravity is balanced with centrifugal force, is given by $R_{c}=j^{2} / G M=p^{2} G M / c_{s}^{2} \sim 250(p / 0.1)^{2}\left(M / 1 M_{\odot}\right) c_{s 190}^{-2} \mathrm{AU}$ [a similar relation is pointed out by Basu (1998)]. This indicates that the centrifugal radius expands with time. On the other hand, a self-similar solution for the rotating isothermal thin disk has been found by Saigo \& Hanawa (1998). Their solution in the accretion phase also shows that the radius where $v_{\phi}$ takes the maximum increases with time as $\propto c_{s} \tau$. Since the outflow is ejected from a radius where $v_{\phi}$ is important (Paper I), these two indicate that the seeding region of the outflow moves outwardly. Using the solution by Saigo \& Hanawa (1998; for an example, see their Figure 7 ), the surface density $(\Sigma)$ and thus the density $(\rho)$ decrease as collapse proceeds and the seeding region moves outwards. This shows that the density near the seeding region decreases as the collapse proceeds.

This leads to the conclusion that the coupling between gas and magnetic fields (Nakano 1990) becomes stronger as long as we consider the seeding region, indicating that the mechanism of angular momentum transfer works also in the later stage of the evolution.

The author thanks R. Ouyed, the referee, for his fruitful comments to improve the paper. He also thanks T. Hanawa, T. Matsumoto, and F. Nakamura for useful discussion. This work was partially supported by Grants-in-Aid from the Ministry of Education, Science, Culture, and Sports (10147105, 11640231). Numerical calculations were performed by Fujitsu VPP300/16 at the Astronomical Data Analysis Center, the National Astronomical Observatory, and NEC SX 4/2B at the Integrated Information Processing Center, Niigata University. 


\section{REFERENCES}

Bachiller, R. 1996, ARA\&A, 34, 111.

Basu, S. 1998, ApJ, 509, 229.

Blandford, R.D., \& Payne, D.G. 1982, MNRAS, 199, 883.

Bouvier, J., Cabrit, C., Fernández, M., Martín, E.L., \& Matthews, J. 1993, A\&A, 272, 176.

Ebert, R., von Hoerner, S., \& Temesvary, S. 1960, Die Entstehung von Sternen durch Kondensation diffuser Materie, (Springer-Verlag, Berlin) p.315.

Fukui, Y., Iwata, T., Mizuno, A., Bally, J. \& Lane, A.P. 1993, in Protostars and Planets III, eds. E.H. Levy, \& J.J. Lunine (Tucson, University Arizona Press) p.603.

Goodman, A.A., Benson, P.J., Fuller, G.A., \& Myers, P.C. 1993, ApJ, 406, 528.

Königl, A. 1989, ApJ, 342, 208.

Kudoh, T., Matsumoto, R., \& Shibata, K. 1998, ApJ, 508, 186.

Larson, R.B. 1969, MNRAS, 145, 271.

Matsumoto, T., Hanawa, T., \& Nakamura, F. 1997, ApJ, 478, 569.

Mouschovias, T.Ch., \&Paleologou, E.V. 1980, ApJ, 237, 877.

Nakano, T. 1990, MNRAS, 242, 535.

Norman, M.L., Wilson, J.R., \& Barton, R.T. 1980, ApJ, 239, 968.

Saigo, K., \& Hanawa, T. 1998, ApJ, 493, 342.

Spitzer, L.Jr. 1978, Physical Processes in the Interstellar Medium (Wiley, New York) §13.3.

Stodółkiewicz, J.S. 1963, Acta Astron., 13, 30.

Tomisaka, K. 1995, ApJ, 438, 226.

Tomisaka, K. 1996a, PASJ, 48, L97. 
Tomisaka, K. 1996b, PASJ, 48, 701.

Tomisaka, K. 1998, ApJ, 502, L163 (Paper I).

Tomisaka, K., Ikeuchi, S., \& Nakamura, T. 1990, ApJ, 362, 202.

Wardle, M., \& Königl, A. 1993, ApJ, 410, 218. 


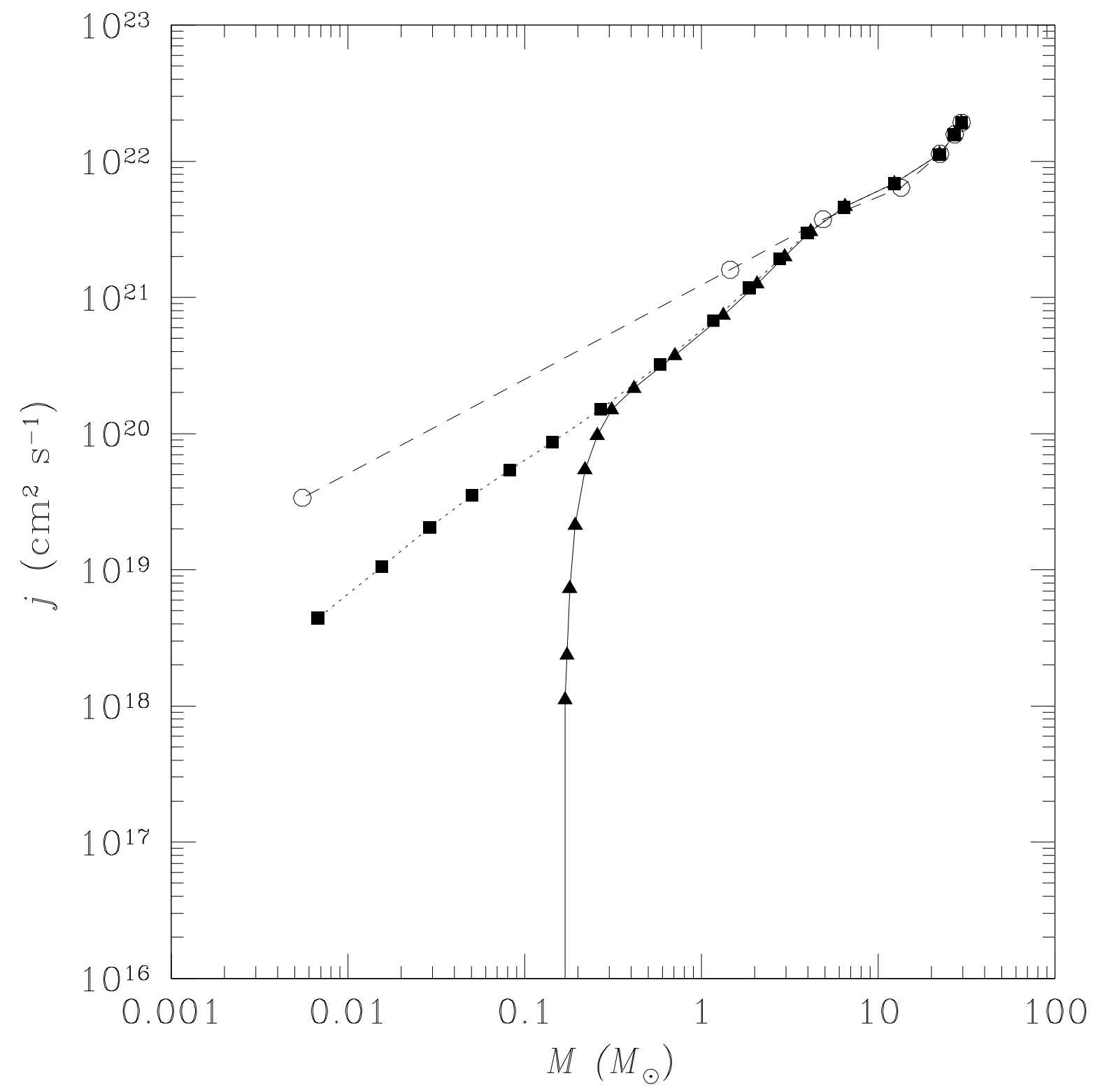

Fig. 1. - Specific angular momentum $j$ is plotted against the accumulated mass from the center $M$. Open circles, filled squares, and triangles denote, respectively, the beginning and the end of the run-away collapse phase and the accretion phase. 


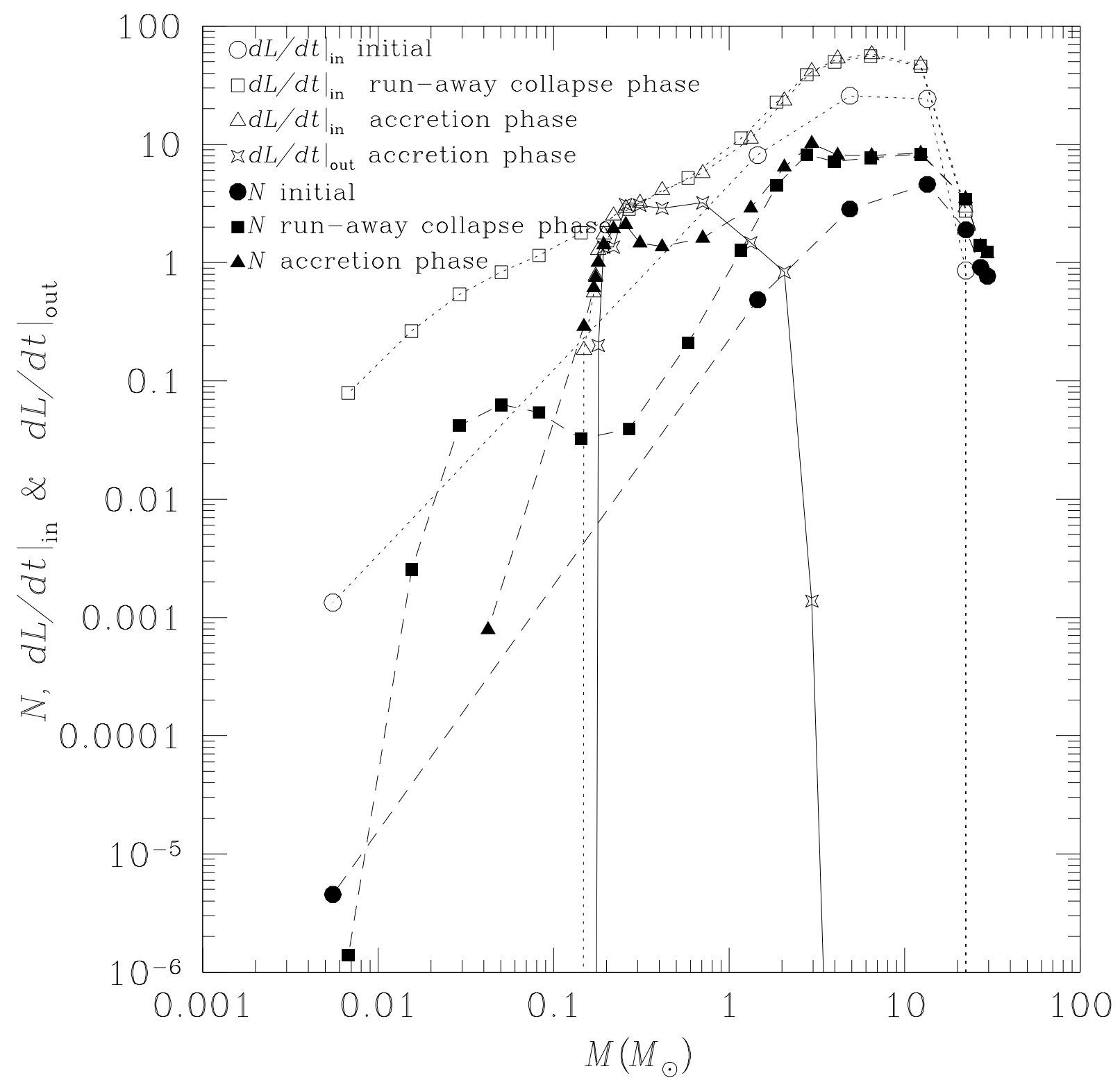

Fig. 2.- The torque exerted on the cloud core by the magnetic fields, $N$, the angular momentum inflow rate convected by the inflow in the disk, $d L /\left.d t\right|_{\text {in }}$, and its outflow rate transferred by the outflow, $d L /\left.d r\right|_{\text {out }}$. Filled and open symbols denote respectively the distribution of $N$ and that of $d L /\left.d t\right|_{\text {in }}$. The circles, and squares denote, respectively, the beginning and the end of the run-away collapse phase. Open stars represent the angular momentum outflow rate $d L /\left.d t\right|_{\text {out }}$ in the accretion phase. 